\title{
Análisis de la percepción que tiene el futuro profesorado sobre el estado actual del mundo
}

Datos de contacto:

Jon Bustillo Bayón

Dpto de Didáctica y

Organización Escolar (UPV/EHU)

Email: jon.bustillo@ehu.eus

Álvaro Antón Baranda

Dpto. de Didáctica de la

Matemática y de las Ciencias

Experimentales (UPV/EHU)

Email: alvaro.anton@ehu.eus

Dirección postal:

Facultad de Educación de Bilbao

Universidad del País Vasco / Euskal

Herriko Unibertsitatea.

Bo Sarriena, s/n

Leioa 48940 (Bizkaia)

\author{
Jon BUSTILLO BAYÓN \\ Álvaro ANTÓN BARANDA
}

\section{RESUMEN}

El presente trabajo analiza el nivel de conocimiento que sobre el mundo actual tienen los futuros docentes que cursan estudios de Grado y Postgrado en Educación en la Universidad del País Vasco. En un contexto de hiperconectividad donde comprobar la veracidad de las informaciones se hace cada vez más complejo, es de gran importancia analizar la percepción que el futuro profesorado tiene sobre el estado actual del mundo. Este conocimiento permite proponer medidas de actuación que ayuden a los docentes a comprender cómo es la adquisición y generación de conocimiento, a través de unos medios de comunicación cada vez más digitalizados. Para ello, se ha utilizado el cuestionario ideado por Rosling et. al, que consta de trece preguntas con tres posibles respuestas cada una. Los resultados obtenidos muestran un porcentaje de aciertos del $21 \%$, una media que no llega a los tres aciertos por persona, y una moda que se sitúa en los dos aciertos. Estos datos son muy similares a los obtenidos por las personas de países socio-económicamente avanzados. Igualmente, existe gran coincidencia en los ítems en los que se obtienen mejores y peores niveles de acierto. El paso por los estudios de educación parece no tener incidencia en el desarrollo de esquemas mentales que ayuden a tener visiones más acertadas de la realidad, que puedan prevenir tanto entre el alumnado como el profesorado la prevalencia de creencias y falacias infundadas.

PALABRAS CLAVE: Formación profesorado; percepción; sesgos cognitivos; confiabilidad; infoxicación; creencias. 


\title{
Analysis of future teachers' perception about the current state of the world
}

\begin{abstract}
This paper analyses the level of knowledge that future teachers, undergraduate and postgraduate students of the University of the Basque Country, have about the current world. In a context of hyper-connectivity, verifying the veracity of information is becoming more and more complex. Thus, it is very important to become aware of the lack of knowledge as the first step in proposing action measures that help teachers to understand what the acquisition and generation of knowledge are like through increasingly digitalized means of communication. For this purpose, the questionnaire designed by Rosling et al was used, which consists of thirteen questions with three possible answers each. The results obtained show a success rate of $21 \%$, an average of less than three correct answers per person, and a mode of two. These data are very similar to those obtained by people from socioeconomically advanced countries. Likewise, there is a great coincidence in the items in which the best and worst levels of success are obtained. Studying education degree studies does not seem to have an impact on the development of mental schemes that help to have more accurate visions of reality, which could avoid the prevalence of unfounded beliefs and fallacies among both students and teachers.
\end{abstract}

KEYWORDS: Teacher training; perception; cognitive biases; factfulness; Infoxication; belief

\section{Introducción}

El desarrollo del conocimiento tiene como compañera inseparable de viaje a la ignorancia, y se podría incluso decir que representan las dos caras de una misma realidad. En este sentido, definir la ignorancia como la ausencia de conocimiento, es en cierta forma acometer un ejercicio de reduccionismo excesivo. La ignorancia no debería entenderse como una falta de habilidades, pericia o inteligencia, sin tener en cuenta fenómenos sociales que condicionan entre otras cuestiones, qué es pertinente saber, qué no, qué es conocible y qué es necesario conocer (High, Kelly, \& Mair, 2012). De hecho, en cada época y contexto ha habido un acuerdo más o menos flexible, que delimitaba aquello que había que saber y aquello que no se debía o podía saber. Cada entorno sociocultural premia una serie de saberes y deja de lado otros. Incluso, entre comunidades diferentes, se pueden observar distintas costumbres que enfocan a unas u otras áreas de conocimiento (High et al., 2012; Croissant, 2014).

En épocas de la historia no tan remotas, el acceso al conocimiento se hacía a través de la transmisión oral o escrita, pero la incorporación de los 
medios de comunicación masivos y muy especialmente, la digitalización de las tecnologías de la información, han dinamitado este modelo. Esto ha incidido en un incremento exponencial de la cantidad de información a la que cada persona está expuesta continuamente, pero en vez de favorecer el acceso a más y mejor información, está actuando en sentido contrario, generando un estado de saturación cognitiva (Schwarzkopf, 2019). Actualmente, cualquier persona con acceso a internet puede publicar lo que considere oportuno, sin que lo que se publique tenga que tener ningún tipo de filtro que permita contrastar la veracidad de la información. Fenómenos como los fakenews o noticias falsas, serian unos de los exponentes de esta anomalia, pero sin ir a estos extremos, existen demasiados sesgos informativos que, de forma más o menos interesada y consciente, solo muestran algunas partes de la realidad que describen (O’Neil, 2017; Lazer et al., 2018; Vosoughi, Roy, \& Aral, 2018). Esto no solo sucede en la propaganda o en los medios de comunicación, es algo que empieza a extenderse a ámbitos tan exigentes como el mundo académico (Chambers, 2017). Autores como Stephens-Davidowitz (2019) ya alertan de la dificultad que implica contrastar la información que se publica en los medios de comunicación digitales, mostrando lo que Ramos Chávez (2018) define como postverdad.

Antes de la popularización de las redes de transmisión de datos a alta velocidad, los medios de comunicación trataban de competir ofreciendo la información más veraz posible, siendo este uno de los principales criterios de calidad. En virtud de la veracidad de la información que publicaban, ciertos medios de comunicación llegaron a ser denominados como "prensa seria". Este mérito se veía reforzado y reconocido por un gran número de lectores, que traian consigo importantes ingresos en forma de un gran volumen de ventas. Esto permitía invertir en más recursos para seguir asegurando que el medio fuera más creíble y rentable. En la actualidad, el modelo de negocio de los medios de comunicación ya no está tan ligado a la calidad de las informaciones, y sí a la captación de la atención de las personas (Davenport \& Beck, 2001; Goldhaber, 2006). En consecuencia, el objetivo último reside en que el mayor número de personas posible acceda a los contenidos incluidos en dichos medios. Desde los servidores que proveen la información se registran los datos relativos a los usuarios, que tras su análisis, permiten descubrir hábitos, costumbres, intereses y características de estas personas (O’Neil, 2017). Una información que actualmente es muy codiciada y que tiene una gran relevancia en las decisiones que se adoptan tanto por parte de las compañias como de las administraciones (Mikalef, Pappas, Krogstie, \& Giannakos, 2018; Popovič, Hackney, Tassabehji, \& Castelli, 2018).

En esta lucha por obtener rentabilidades económicas, las compañias especializadas en comunicación, dedican gran cantidad de recursos a explotar los sesgos cognitivos. Esto permite favorecer el incremento del periodo medio de conexión, y del número de veces que cada usuario vuelve 
a un determinado medio (Albarran, 2016; Baeza-Yates, 2018). Las compañias que no atiendan a estas dos claves tienen muy dificil competir por la atención del público general, perdiendo competitividad frente a otros medios que sí los tienen en consideración (Fisher, 2015).

Explotar estos sesgos cognitivos facilita que, muchas personas tomen como ciertas informaciones no contrastadas o sesgadas, comenzando así a formar opiniones infundadas especialmente sobre temas complejos que son tratados con regularidad en los medios de comunicación. Este tipo de contenidos suelen tratar sobre la evolución de la demografia, la pobreza, el cambio climático, la delincuencia, la violencia, etc. Y, el tratamiento que reciben estos temas suele estar asociado a circunstancias llamativas, que son muy útiles para captar con rapidez la atención de las personas. Se seleccionan informaciones y formatos impactantes, independientemente de que se ajusten más o menos a la realidad. Trabajos como los realizados por Lazer et al., (2018) y Vosoughi et al., (2018) encontraron que las noticias falsas tienen tendencia a perdurar más en los medios, que las que se ajustan a la realidad, cuestión que agrava, más si cabe, la difícil tarea de distinguir entre aquello que es cierto y lo que no lo es.

En la actualidad, más del $85 \%$ de las personas tienen acceso a internet en el estado español (We are social, 2019) y esto implica que la mayoría de la sociedad está procesando de forma continuada, grandes cantidades de informaciones en formatos multimedia que abordan temas de lo más diversos. Se podría decir que el acceso a la información nunca fue tan sencillo, y es por ello que, cabría imaginar una sociedad más y mejor informada sobre lo que acontece en el mundo en el que vivimos (Mayos Solsona, Brey, \& Innerarity, 2011). La realidad parece ser otra, y aunque efectivamente en la actualidad hay acceso a informaciones que, en otras épocas simplemente no eran accesibles; la interpretación que de estas se realiza no siempre ofrece una imagen que se ajuste a la realidad. Procesar tal cantidad de información y especialmente sobre temas complejos no es tarea sencilla, máxime cuando la velocidad con la que esta se presenta es más elevada de lo que el cerebro humano necesita para su análisis (Carr, 2010). Saber distinguir dentro del gran flujo de información, qué se ajusta a la realidad y qué no, se está convirtiendo en un ejercicio muy complejo que requiere un esfuerzo demasiado grande (Greer, 2003; Dias, 2014; Yarritu, Matute, \& Luque, 2015; Blanco \& Matute, 2018). Esta dificultad para identificar aquella información que no es fidedigna, es una preocupación que ya es manifestada por más del 69\% de las personas internautas (Amoedo, Vara-Miguel, \& Negredo, 2018).

La exposición continuada a permanentes flujos de información favorece el desarrollo de una falsa sensación de conocimiento. Trabajos anteriores han mostrado que las personas con menos conocimientos tienden a evaluarse a sí mismas, como mucho más expertas de lo que su nivel de conocimientos indica (Dunning, 2011; Atir, Rosenzweig, \& Dunning, 2015; Anson, 2018). Este fenómeno conocido como efecto "Dunnig-Kruger", trae 
consigo una falsa sensación de dominio sobre temáticas de las que apenas se tiene conocimientos, lo que conlleva a que no se perciba la necesidad de profundizar y analizar con más detalle o rigor. Esta situación es particularmente preocupante con unas informaciones que cada vez están más sesgadas para que estas se ajusten a las ideologías e intereses de las personas, favoreciendo así que se destine la atención hacia aquellos medios, o mensajes, que presentan la información conforme a las creencias e intereses de cada persona (Casselman, 2011; Danovitch, 2019). Ante esta incesante recepción de información, la atención se centra preferentemente sobre aquellas temáticas y enfoques que más se acercan a la forma de interpretar el mundo de cada receptor, (independientemente de que se ajusten o no a la realidad), reforzando así la sensación de conocimiento. Esto reafirma nuevamente las ideas previas, y estas vuelven a dar una falsa sensación de conocimiento. Un círculo vicioso que favorece la propagación de la ignorancia y del que no es sencillo salir (Croissant, 2014).

Comprender cómo se dan estos procesos de adquisición de información masiva y continuada a través de las diferentes fuentes de información, puede ser de gran ayuda. Especialmente para las generaciones venideras, y en este sentido la Escuela tiene una posición muy relevante, ya que ahí se decide qué y qué no deben saber las personas en edad escolar. Una selección de conocimientos y habilidades (hoy enmascaradas bajo el paraguas de las competencias) pretenden servir de base común a las personas pertenecientes a una comunidad cada vez más global. Quizás, una de las preguntas clave es determinar si es o no importante y relevante que el profesorado que actúa como docente en los cursos de educación obligatoria (por donde pasa casi el 100\% de la población en edad escolar), tenga el hábito de utilizar mecanismos que le permitan discriminar entre las informaciones más veraces y las que lo son menos. En un primer momento parece claro que sería deseable un cuerpo docente que filtre informaciones que estén sesgadas y que no ofrezcan una visión que se ajuste a la realidad (Garrett \& Segall, 2013). Esto permitiría que el alumnado pueda tener modelos de personas que utilizan mecanismos cognitivos para filtrar con mayores garantias la información a la que constantemente estarán expuestos.

En el análisis de cómo se da el desarrollo del pensamiento crítico, autores como Schommer (1993, 1998) y Perry (1998) proponen esquemas con varias etapas que se van cumpliendo de forma secuencial, pasando de un pensamiento dual a uno relativista, en donde el uso de la razón y la evaluación de los diferentes puntos de vista adquieren una relevancia cada vez mayor a la hora de interpretar la información. No obstante, otros autores como Magolda (2006), Richardson, (2013) y Ouellette-Schramm (2015) además de destacar la relevancia del recorrido epistemológico individual, hacen referencia a la necesidad de considerar otros factores como la concepción del aprendizaje, las creencias sobre las fuentes de información, el entorno social y los procesos de maduración. 
Tal y como indican Rosling et al. (2018) el problema no reside tanto en que no se acceda a la información correcta, cómo que una multitud distribuida por diferentes países tenga visiones equivocadas del mundo. En cierta forma, esto indica la existencia de una idea incorrecta compartida por miles de personas, que como es previsible, provienen de contextos culturales diferentes y, por tanto, no han accedido a las mismas fuentes informativas. Este tipo de errores o sesgos cognitivos han sido bien estudiados en trabajos previos (Tversky \& Kahneman, 1974; Gilovich \& Griffin, 2010; Kahneman, 2012; Kahneman \& Tversky, 2013; Yarritu et al., 2015; Kaufman \& Kaufman, 2018) y muestran cómo existen algunas tendencias comunes a la hora de interpretar el entorno y la información que se deriva de él. En muchos de los casos este análisis se realiza de forma rápida e intuitiva, un modelo que en el proceso de desarrollo de nuestra sociedad hasta hoy en día ha resultado de gran valor, pero que en las condiciones actuales nos lleva a interpretar incorrectamente la información a la que accedemos (Slovic, Peters, Finucane, \& MacGregor, 2005; Yarritu et al., 2015).

\section{Método}

En este trabajo se pretende medir el grado de conocimiento que sobre el mundo tiene una muestra representativa de las personas que en los próximos años empezarán a ejercer como docentes en las aulas de educación obligatoria en la Comunidad Autónoma del País Vasco. Para ello se va a replicar el test diseñado por Rosling et al. (2018), que hace preguntas generales sobre el estado actual del mundo. En esta prueba se realizan trece preguntas con tres respuestas posibles que pretenden medir no tanto el dato concreto, sino la perspectiva que se tiene frente a temas que son abordados en los medios de comunicación de forma recurrente. Para ello se trabajan cuestiones asociadas a la pobreza, al cambio climático, a la evolución demográfica, a la esperanza de vida, a los desastres naturales, al acceso a electricidad, a la escolarización, a la vacunación y a la conservación de la naturaleza. Para desarrollar este trabajo se ha determinado hacer el estudio sobre una muestra que al 95\% sea representativa, del colectivo de personas que actualmente están desarrollando estudios para acceder al cuerpo de docentes, de las etapas de educación Primaria y Secundaria obligatoria en las tres Facultades de Educación de la Universidad del País Vasco / Euskal Herriko Unibertsitatea (en adelante UPV/EHU). Con un universo de 1603 alumnos entre los Grados de Educación Primaria y el Máster de Educación Secundaria, se ha utilizado una muestra de 369 estudiantes en el curso lectivo 2018-2019.

El cuestionario y la metodología cumplen con la normativa vigente y cuentan con el visto bueno del comité de ética de la UPV/EHU (CEISHUPV/EHU, Ref. M10_2019_127). Se ha utilizado un formulario online para 
favorecer la voluntariedad y que garantiza el anonimato.

El cuestionario consta de tres apartados: el primero de información y que incluye el consentimiento informado para el uso en investigación de los datos generados. Seguidamente se pregunta el nivel de estudios terminados para poder organizar las respuestas en función del grado de formación. Por último, se realiza el cuestionario con trece preguntas que van apareciendo en orden aleatorio, disponiendo de un tiempo máximo de 15 minutos para completar la prueba.

En el análisis de los resultados se han realizado medias, desviaciones típicas y modas de las respuestas recogidas por cada colectivo, para comparar los resultados con los de estudios previos. Igualmente, se ha estudiado si existen diferencias relevantes entre las personas que están cursando estudios de Grado frente a las de Postrgado. Se ha utilizado el paquete estadistico de Microsoft Excel.

\section{Resultados}

Sobre un universo de 1603 personas, se obtuvieron 369 respuestas. De las cuales 237 corresponden a estudiantes de Grado de Educación Primaria y 132 a estudiantes del Máster de Secundaria. La media de aciertos es de 2.75 sobre 13 , con una desviación típica de 1.51 y una moda de 2 aciertos. Desglosando los resultados entre el nivel académico, los pregraduados obtienen una media de 2.93 sobre 13, con una desviación típica de 1.53 y una moda y mediana de 3 aciertos. Por el contrario, las personas ya graduadas obtienen una media de 2.44 sobre 13 , una desviación típica de 1.42 y una moda y mediana de 2 aciertos. Las diferencias observadas son estadísticamente significativas (Kruskal-Wallis, prueba no paramétrica $\mathrm{P}=0,001)$.

En la tabla 1 se recogen las preguntas del cuestionario, así como el porcentaje de aciertos obtenidos para cada grupo académico. Se incluyen además los datos obtenidos por el trabajo de Rosling et al., (2018).

Tabla 1

Porcentaje de acierto asociado a cada pregunta

\begin{tabular}{|l|c|c|c|c|c|}
\hline Preguntas & Total & Pregrado & Postgrado & Factfulness & España \\
\hline $\begin{array}{l}\text { En los últimos 20 años, la } \\
\text { proporción de personas que viven } \\
\text { en la pobreza extrema en todo el } \\
\text { mundo, ha...? }\end{array}$ & $6 \%$ & $7 \%$ & $3 \%$ & $9 \%$ & $3 \%$ \\
\hline $\begin{array}{l}\text { ¿Cuántos de los niños de 1 año } \\
\text { de edad de todo el mundo han } \\
\text { sido vacunados contra alguna } \\
\text { enfermedad? }\end{array}$ & $8 \%$ & $8 \%$ & $6 \%$ & $13 \%$ & $13 \%$ \\
\hline $\begin{array}{c}\text { ¿Cómo ha cambiado el número } \\
\text { de muertes por año a causa de }\end{array}$ & $19 \%$ & $22 \%$ & $15 \%$ & $10 \%$ & $9 \%$ \\
\hline
\end{tabular}


Jon Bustillo Bayón y Álvaro Antón Baranda

\begin{tabular}{|c|c|c|c|c|c|}
\hline $\begin{array}{l}\text { desastres naturales en los } \\
\text { últimos cien años? }\end{array}$ & & & & & \\
\hline $\begin{array}{l}\text { ¿Dónde vive la mayoría de la } \\
\text { población mundial? }\end{array}$ & $22 \%$ & $23 \%$ & $20 \%$ & $26 \%$ & $24 \%$ \\
\hline $\begin{array}{l}\text { En todo el mundo, los hombres } \\
\text { de } 30 \text { años han pasado } 10 \text { años } \\
\text { en la escuela, en promedio. } \\
\text { ¿Cuántos años han pasado las } \\
\text { mujeres de la misma edad en } \\
\text { la escuela? }\end{array}$ & $13 \%$ & $12 \%$ & $14 \%$ & $20 \%$ & $13 \%$ \\
\hline $\begin{array}{l}\text { Hay aproximadamente siete mil } \\
\text { millones de personas en el } \\
\text { mundo hoy en día. ¿Qué mapa } \\
\text { muestra dónde vive la gente? } \\
\text { (Cada cifra representa a mil } \\
\text { millones de personas.) }\end{array}$ & $13 \%$ & $11 \%$ & $18 \%$ & $28 \%$ & $23 \%$ \\
\hline $\begin{array}{l}\text { Las Naciones Unidas pronostican } \\
\text { que para el año } 2100 \text { la } \\
\text { población mundial habrá } \\
\text { aumentado en otros } 4.000 \\
\text { millones de personas. ¿Cuál es } \\
\text { la razón principal? }\end{array}$ & $20 \%$ & $25 \%$ & $10 \%$ & $26 \%$ & $26 \%$ \\
\hline $\begin{array}{l}\text { En los países de bajos ingresos } \\
\text { de todo el mundo, ¿cuántas } \\
\text { niñas terminan el quinto } \\
\text { grado? }\end{array}$ & $3 \%$ & $4 \%$ & $2 \%$ & $7 \%$ & $4 \%$ \\
\hline $\begin{array}{l}\text { Hoy en día hay } 2.000 \text { millones } \\
\text { de niños en el mundo, de } 0 \text { a } \\
15 \text { años de edad. ¿Cuántos } \\
\text { niños habrá en el año } 2100 \\
\text { según las Naciones Unidas? }\end{array}$ & $34 \%$ & $37 \%$ & $29 \%$ & $15 \%$ & $21 \%$ \\
\hline $\begin{array}{l}\text { ¿Cuántas personas en el mundo } \\
\text { tienen acceso a la electricidad? }\end{array}$ & $19 \%$ & $21 \%$ & $15 \%$ & $22 \%$ & $14 \%$ \\
\hline $\begin{array}{l}\text { ¿Cuál es la esperanza de vida de } \\
\text { la población mundial? }\end{array}$ & $20 \%$ & $24 \%$ & $14 \%$ & $37 \%$ & $41 \%$ \\
\hline $\begin{array}{l}\text { Los tigres, pandas gigantes y } \\
\text { rinocerontes negros fueron } \\
\text { incluidos en la lista de especies } \\
\text { amenazadas en 1996. Desde } \\
\text { entonces, ¿alguna de estas } \\
\text { especies se ha vuelto más } \\
\text { amenazada? }\end{array}$ & $4 \%$ & $4 \%$ & $3 \%$ & $9 \%$ & $7 \%$ \\
\hline $\begin{array}{l}\text { Los expertos en clima global } \\
\text { creen que, en los próximos } 100 \\
\text { años, la temperatura media lo } \\
\text { hará: }\end{array}$ & $95 \%$ & $95 \%$ & $95 \%$ & $87 \%$ & $92 \%$ \\
\hline ACIERTOS TOTALES & $21 \%$ & $23 \%$ & $19 \%$ & $18 \%$ & $17 \%$ \\
\hline
\end{tabular}


En la tabla2 se presenta la distribución de la muestra en función del número de aciertos.

Tabla 2

Porcentaje de personas por número de aciertos

\begin{tabular}{|c|c|c|c|c|c|c|c|c|c|c|c|c|c|c|}
\hline & 0 & 1 & 2 & 3 & 4 & 5 & 6 & 7 & 8 & 9 & 10 & 11 & 12 & 13 \\
\hline Pregrado & $1 \%$ & $20 \%$ & $22 \%$ & $22 \%$ & $20 \%$ & $9 \%$ & $5 \%$ & $0 \%$ & $1 \%$ & $0 \%$ & $0 \%$ & $0 \%$ & $0 \%$ & $0 \%$ \\
\hline Postgrado & $0 \%$ & $27 \%$ & $36 \%$ & $17 \%$ & $11 \%$ & $6 \%$ & $2 \%$ & $0 \%$ & $2 \%$ & $0 \%$ & $0 \%$ & $0 \%$ & $0 \%$ & $0 \%$ \\
\hline TOTAL & $1 \%$ & $23 \%$ & $27 \%$ & $20 \%$ & $17 \%$ & 85 & $4 \%$ & $0 \%$ & $1 \%$ & $0 \%$ & $0 \%$ & $0 \%$ & $0 \%$ & $0 \%$ \\
\hline
\end{tabular}

\section{Discusión}

Los resultados del presente trabajo son coincidentes con los obtenidos por Rosling et al. (2018) y muestran que el alumnado de las Facultades de Educación de la UPV/EHU tiene una idea del mundo, igual de distorsionada que la mayor parte de la población que vive en contextos socioeconómicos similares. El futuro profesorado ha tenido que completar una larga vida académica adquiriendo un grado de educación mayor que la muestra del trabajo replicado. Sin embargo, en los planes formativos del profesorado no hay asignaturas que de forma específica trabajen cómo son los procesos de generación de conocimiento en entornos hiperconectados. Por ello, la imagen que sobre el mundo tienen las personas de esta muestra, se corresponde con una visión ampliamente compartida socialmente y que no implica ningún tipo de disonancia con respecto a lo que se piensa y se transmite en la mayoría de las familias de los escolares. Los estudios de Grado y Postgrado educativos no están realizando una corrección y en este sentido, es preciso destacar que el número de aciertos de las personas postgraduadas, es ligeramente menor que el de las personas que están actualmente cursando el Grado (23\% frente a $19 \%$ de aciertos). Según estos resultados, la acumulación de cursos orientados hacia la profesión docente, no solo no asegura una mejora, sino que está implicando un empeoramiento en el análisis del mundo actual. Coincidiendo con Viskontas (2018) se podría hablar de un proceso de polarización, reafirmando sus creencias erróneas. De hecho, la gran mayoría de las personas que residen en los países del primer mundo, a pesar de haber tenido la posibilidad de realizar estudios mínimos de secundaria, muestran un conocimiento muy pobre sobre temas que son habitualmente tratados de forma sesgada en los medios de comunicación. Incluso, cuando se ha realizado el mismo test a responsables y asesores participantes en el Foro Económico Mundial de Davos (2015), la tasa de aciertos apenas llegó al 18\%.

Entre las preguntas realizadas hay una que destaca por haber sido respondida correctamente por más del $95 \%$ de las personas participantes. Se refiere al efecto que sobre la temperatura del planeta está teniendo el 
cambio climático. Hay que señalar que, al igual que sucede en los ítems en donde la tasa de aciertos es menor, el porcentaje de aciertos es muy similar al de las muestras globales. El desconocimiento adquiere así una perspectiva social y evolutiva, especialmente a la hora de abordar problemas complejos como los temas tratados en el cuestionario de este trabajo.

En la actualidad, la Escuela tiene muy dificil competir con los canales multimedia, que a través de una gran variedad de dispositivos muy llamativos y accesibles, están ofreciendo información no contrastada de forma ininterrumpida (Sarramona, 1986; Caro-Samada, 2015). Este flujo continuo y complejo, favorece la aparición y el uso de esquemas mentales más o menos automatizados para procesar la información recibida. Incluso aunque las conclusiones que se deriven de su interpretación no se ajusten con la realidad (Tetlock \& Gardiner, 2017). Por ello, resulta determinante ahondar en el conocimiento del modo en que las personas interpretan lo que reciben de los medios de información (Dias, 2014; Yarritu et al., 2015).

Los sesgos cognitivos han contribuido al desarrollo evolutivo de las personas, premiando la eficacia frente al conocimiento de la verdad. Realizar estimaciones, incluso aunque en ocasiones no sean correctas, no siempre implica una mejora evolutiva y sí un coste cognitivo muy elevado (Tversky \& Kahneman, 1974; Matute, Yarritu, \& Vadillo, 2011; Blanco \& Matute, 2018). El coste de asumir algunas respuestas equivocadas a situaciones que no revierten peligro, sería un mal mucho menor comparado con la no respuesta a situaciones peligrosas no detectadas (Matute et al., 2011). Este proceso, además de por cuestiones de economía cognitiva, vendría reforzado por una pertenencia social en donde se comparte una interpretación de la realidad a través de fuentes y sesgos similares.

En el caso de este trabajo, las respuestas han sido recogidas con una limitación de tiempo que impedía que las personas participantes se pudieran documentar de fuentes externas. Habia que dar la mejor respuesta posible a través de las creencias e informaciones derivadas de la visión del mundo que cada participante tenía, y que como se ha podido comprobar, está distorsionada. Pretender que el colectivo docente minimice las visiones distorsionadas que sobre el mundo pudiera tener, sería un escenario deseable. No obstante, hay que tener presente la influencia que tienen los rápidos cambios que se dan en esta sociedad hiperconectada, afectando al volumen y a la calidad de las informaciones a las que las personas se ven expuestas, y con ello, moldeando de forma permanente los limites de la ignorancia (Weinberger, 2011). En la medida en que el conocimiento crece, lo desconocido aumenta (Dunning, 2011; Croissant, 2014; Frazier, 2015).

Convivir con la ignorancia es algo que lejos de justificar el desconocimiento, debería ser visto como un motor del conocimiento. Desde esta perspectiva obtener consciencia sobre el desconocimiento, abre la puerta al aprendizaje (Tetlock \& Gardiner, 2017). Comprender los motivos 
por los que las personas, después de largos periodos formativos, siguen teniendo visiones distorsionadas de las realidades cotidianas, es un buen comienzo para entender cómo se genera y adquiere el conocimiento. Trabajos previos han mostrado que los sesgos cognitivos pueden reducirse a través de intervenciones educativas que enseñan a escolares técnicas para obtener una visión más realista de lo que les muestran las informaciones recibidas desde los diferentes medios (Barberia, Blanco, Cubillas, \& Matute, 2013; Barberia, Tubau, Matute, \& Rodriguez-Ferreiro, 2018). Esta idea coincide con los trabajos de Shirky $(2010,2011)$ que pone el acento en la dificultad de realizar unos filtrados de la información que sean realmente efectivos. En este sentido, Rosling et al. (2018) proponen que se enseñen en las escuelas unas reglas generales para que la mayoría del alumnado logre, a través de la información a la que estará expuesto, una visión más ajustada a la realidad. Estas iniciativas favorecerian el desarrollo de un pensamiento crítico que permita a las generaciones más jóvenes, lo que Delors (1997) definió como "aprender a conocer", uno de los cuatro pilares básicos de su ampliamente aceptada y extendida propuesta educativa.

Si la imagen del mundo que tienen los docentes, aun no siendo acertada, se ajusta a la que tiene su entorno, no se percibe la necesidad de que adquieran formación relativa a la incidencia que los sesgos cognitivos tienen a la hora de interpretar la realidad. Sin embargo, los resultados obtenidos en el presente trabajo reflejan la necesidad de incorporar en la formación de los futuros docentes actuaciones que les permitan ser conscientes de sus limitaciones a la hora de interpretar temas complejos. Desde esta nueva perspectiva, el alumnado estaría más receptivo para entender cómo son los mecanismos que le han llevado a la asimilación, por hiper exposición a los medios de comunicación actuales, de estas creencias infundadas y ampliamente compartidas socialmente.

\section{Referencias}

Albarran, A. B. (2016). The Media Economy (2.a ed.). https://doi.org/10.4324/9781315715094

Amoedo, A., Vara-Miguel, A., \& Negredo, S. (2018). Digital News Report 2018 (p. 81). Recuperado de Universidad de Navarra website: https://bit.ly/2H5h5r8

Anson, I. G. (2018). Partisanship, Political Knowledge, and the DunningKruger Effect: Partisanship, Political Knowledge, and the DunningKruger Effect. Political Psychology, 39(5), 1173-1192. https://doi.org/10.1111/pops. 12490

Atir, S., Rosenzweig, E., \& Dunning, D. (2015). When Knowledge Knows No Bounds: Self-Perceived Expertise Predicts Claims of Impossible Knowledge. Psychological Science, 26(8), 1295-1303. 
https://doi.org/10.1177/0956797615588195

Baeza-Yates, R. (2018). Bias on the web. Communications of the ACM, 61(6), 54-61. https://doi.org/10.1145/3209581

Barberia, I., Blanco, F., Cubillas, C. P., \& Matute, H. (2013). Implementation and Assessment of an Intervention to Debias Adolescents against Causal Illusions. PLoS ONE, 8(8), e71303. https://doi.org/10.1371/journal.pone.0071303

Barberia, I., Tubau, E., Matute, H., \& Rodríguez-Ferreiro, J. (2018). A short educational intervention diminishes causal illusions and specific paranormal beliefs in undergraduates. PLOS ONE, 13(1), e0191907. https://doi.org/10.1371/journal.pone.0191907

Blanco, F., \& Matute, H. (2018). The illusion of causality: A cognitive bias underlying pseudoscience. En Pseudoscience: The conspiracy against science. (pp. 45-75). Cambridge, MA, US: MIT Press.

Caro-Samada, M. del C. (2015). Información y verdad en el uso de las redes sociales por parte de adolescentes. Teoría de la educación, 27(1), 187199. https://dx.doi.org/10.14201/teri.13081

Carr, N. G. (2010). The shallows: how the internet is changing the way we think, read and remember. London: Atlantic.

Casselman, A. (2011). The Google Effect. Scientific American Mind, 22(6), 7 7. https://doi.org/10.1038/scientificamericanmind0112-7b

Chambers, C. (2017). The seven deadly sins of psychology: a manifesto for reforming the culture of scientific practice. Princeton: Princeton University Press.

Croissant, J. L. (2014). Agnotology: Ignorance and Absence or Towards a Sociology of Things That Aren't There. Social Epistemology, 28(1), 425. https://doi.org/10.1080/02691728.2013.862880

Danovitch, J. H. (2019). Growing up with Google: How children's understanding and use of internet- based devices relates to cognitive development. Human Behavior and Emerging Technologies, 1(2), 8190. https://doi.org/10.1002/hbe2.142

Davenport, T. H., \& Beck, J. C. (2001). The attention economy: understanding the new currency of business. Boston, Mass: Harvard Business School Press.

Delors, J. (1997). La educación encierra un tesoro. México: Unesco.

Dias, P. (2014). From 'infoxication' to 'infosaturation': a theoretical overview of the cognitive and social effects of digital immersion. Ámbitos. Revista Internacional de Comunicación, 24(1), 1-11.

Dunning, D. (2011). The Dunning-Kruger Effect. En Advances in Experimental Social Psychology (Vol. 44, pp. 247-296). https://doi.org/10.1016/B978-0-12-385522-0.00005-6 
Fisher, E. (2015). 'You Media': audiencing as marketing in social media. Media, Culture \& Society, 37(1), 50-67. https://doi.org/10.1177/0163443714549088

Frazier, T. Z. (2015). Agnotology and information: Agnotology and Information. Proceedings of the Association for Information Science and Technology, $52(1)$,

$1-3$. https://doi.org/10.1002/pra2.2015.1450520100123

Garrett, H. J., \& Segall, A. (2013). (Re)Considerations of Ignorance and Resistance in Teacher Education. Journal of Teacher Education, 64(4), 294-304. https://doi.org/10.1177/0022487113487752

Gilovich, T. D., \& Griffin, D. W. (2010). Judgment and Decision Making. En S. T. Fiske, D. T. Gilbert, \& G. Lindzey (Eds.), Handbook of Social Psychology (pp.

542-588).

https://doi.org/10.1002/9780470561119.socpsy001015

Goldhaber, M. (2006). The value of openness in an attention economy. First Monday, 11(6). https://doi.org/10.5210/fm.v11i6.1334

Greer, J. D. (2003). Evaluating the Credibility of Online Information: A Test of Source and Advertising Influence. Mass Communication and Society, 6(1), 11-28. https://doi.org/10.1207/S15327825MCS0601_3

High, C., Kelly, A. H., \& Mair, J. (Eds.). (2012). The Anthropology of Ignorance. https://doi.org/10.1057/9781137033123

Kahneman, D. (2012). Thinking, fast and slow. London: Penguin Books.

Kahneman, D., \& Tversky, A. (2013). Choices, Values, and Frames. En L. C. MacLean \& W. T. Ziemba, World Scientific Handbook in Financial Economics Series (Vol. 4, pp. 269-278). https://doi.org/10.1142/9789814417358_0016

Kaufman, A. B., \& Kaufman, J. C. (Eds.). (2018). Pseudoscience (Vol. 1). https://doi.org/10.7551/mitpress/9780262037426.001.0001

Lazer, D. M. J., Baum, M. A., Benkler, Y., Berinsky, A. J., Greenhill, K. M., Menczer, F., ... Zittrain, J. L. (2018). The science of fake news. Science, 359(6380), https://doi.org/10.1126/science.aao2998

Magolda, M. B. B. (2006). Intellectual Development in the College Years. Change: The Magazine of Higher Learning, 38(3), 50-54. https://doi.org/10.3200/CHNG.38.3.50-54

Matute, H., Yarritu, I., \& Vadillo, M. A. (2011). Illusions of causality at the heart of pseudoscience: Illusions of causality in pseudoscience. British Journal of Psychology, 102(3), 392-405. https://doi.org/10.1348/000712610X532210

Mayos Solsona, G., Brey, A., \& Innerarity, D. (2011). La sociedad de la ignorancia (1. ed). Barcelona: Ediciones Península. 
Mikalef, P., Pappas, I. O., Krogstie, J., \& Giannakos, M. (2018). Big data analytics capabilities: a systematic literature review and research agenda. Information Systems and e-Business Management, 16(3), 547578. https://doi.org/10.1007/s10257-017-0362-y

O’Neil, C. (2017). Armas de destrucción matemática: Cómo el bigdata aumenta la desigualdad y amenaza la democracia. Madrid: Capitán Swing Libros.

Ouellette-Schramm, J. (2015). Epistemological development and critical thinking in post secondary. Australian Journal of Adult Learning, 55(1), 114-134.

Perry, J. W. G. (1998). Forms of Intellectual and Ethical Development in the College Years: A Scheme. New Jersey: Wiley.

Popovič, A., Hackney, R., Tassabehji, R., \& Castelli, M. (2018). The impact of big data analytics on firms' high value business performance. Information Systems Frontiers, 20(2), 209-222. https://doi.org/10.1007/s10796-016-9720-4

Ramos Chávez, A. (2018). Información líquida en la era de la posverdad. Revista General de Información y Documentación, 28(1), 275-282. https://doi.org/10.5209/RGID.60809

Richardson, J. T. E. (2013). Epistemological development in higher education. Educational Research Review, 9, 191-206. https://doi.org/10.1016/j.edurev.2012.10.001

Rosling, H., Rosling, O., \& Rönnlund, A. R. (2018). Factfulness: ten reasons we're wrong about the world and why things are better than you think. London: Sceptre.

Sarramona, J. (1986). Influencia de los "mass media" sobre la escuela. Teoría de la educación, 29-44. https://dx.doi.org/10.14201/teri.2810

Schommer, M. (1993). Epistemological development and academic performance among secondary students. Journal of Educational Psychology, 85(3), 406-411. https://doi.org/10.1037/00220663.85.3.406

Schommer, M. (1998). The influence of age and education on epistemological beliefs. British Journal of Educational Psychology, 68(4), 551-562. https://doi.org/10.1111/j.20448279.1998.tb01311.x

Schwarzkopf, S. (2019). Sacred Excess: Organizational Ignorance in an Age of Toxic Data. Organization Studies, 1-21. https://doi.org/10.1177/0170840618815527

Shirky, C. (2010). Cognitive Surplus: How Technology Makes Consumers into Collaborators. New York: Penguin Publishing Group.

Shirky, C. (2011). The Political Power of Social Media: Technology, the 
Public Sphere, and Political Change. Foreign Affairs, 90(1), 28-41. https://www.jstor.org/stable/25800379

Slovic, P., Peters, E., Finucane, M. L., \& MacGregor, D. G. (2005). Affect, risk, and decision making. Health Psychology, 24, S35-S40. https://doi.org/10.1037/0278-6133.24.4.S35

Stephens-Davidowitz, S. (2019). Todo el mundo miente: Lo que internet y el Big Data puede decirnos sobre nostros mismos. Madrid: CAPITAN SWING.

Tetlock, P. E., \& Gardiner, D. (2017). Superpronosticadores: El arte y la ciencia de la predicción. Madrid: Katz editores.

Tversky, A., \& Kahneman, D. (1974). Judgment under Uncertainty: Heuristics and Biases. Science, 185(4157), 1124-1131. https://doi.org/10.1126/science.185.4157.1124

Viskontas, I. (2018). The Challenges of Changing Minds: How Confirmation Bias and Pattern Recognition Affect Our Search for Meaning. En A. B. Kaufman \& J. C. Kaufmann, Pseudoscience: The Conspiracy Against Science. Massachusetts: The MIT Press.

Vosoughi, S., Roy, D., \& Aral, S. (2018). The spread of true and false news online. Science, 359(6380), 1146-1151. https://doi.org/10.1126/science.aap9559

We are social. (2019). We are social, 2019 (España). Recuperado de We are social website: https:// bit.ly/2MNyJyO

Weinberger, D. (2011). Too big to know: rethinking knowledge now that the facts aren't the facts, experts are everywhere, and the smartest person in the room is the room. New York: Basic Books.

Yarritu, I., Matute, H., \& Luque, D. (2015). The dark side of cognitive illusions: When an illusory belief interferes with the acquisition of evidence-based knowledge. British Journal of Psychology, 106(4), 597608. https://doi.org/10.1111/bjop.12119 
